Review Article

\title{
Murdannia loriformis: A Review of Ethnomedicinal Uses, Phytochemistry, Pharmacology, Contemporary Application, and Toxicology
}

\author{
Intan Soraya Che Sulaiman ${ }^{\mathbb{D}},{ }^{1}$ Azham Mohamad ${ }^{\mathbb{D}},{ }^{2}$ and Osumanu Haruna Ahmed ${ }^{30,4}$ \\ ${ }^{1}$ Faculty of Applied Sciences, Universiti Teknologi Mara, Perlis Branch, Arau Campus, Shah Alam 02600, Perlis, Malaysia \\ ${ }^{2}$ Centre of Foundation Studies for Agricultural Science, Universiti Putra Malaysia, Serdang 43400, Selangor, Malaysia \\ ${ }^{3}$ Department of Crop Science, Faculty of Agriculture and Food Sciences, Universiti Putra Malaysia Bintulu Campus, \\ Bintulu 97008, Sarawak, Malaysia \\ ${ }^{4}$ Institut Ekosains Borneo, Universiti Putra Malaysia Bintulu Campus, Bintulu 97008, Sarawak, Malaysia \\ Correspondence should be addressed to Azham Mohamad; azham007@yahoo.com
}

Received 21 March 2021; Accepted 25 June 2021; Published 6 July 2021

Academic Editor: Daniel Dias Rufino Arcanjo

Copyright (c) 2021 Intan Soraya Che Sulaiman et al. This is an open access article distributed under the Creative Commons Attribution License, which permits unrestricted use, distribution, and reproduction in any medium, provided the original work is properly cited.

\begin{abstract}
This review provides an updated and comprehensive overview on the ethnomedicinal use, phytochemistry, pharmacology, and toxicology of $M$. loriformis. Phytochemical analysis of $M$. loriformis revealed that it is composed of phenolics, flavonoids, condensed tannins, chlorophylls, alkaloids, and steroids. Numerous compounds including syringic acid, $\beta$-O-D-glucopyranosyl2 -(2'-hydroxy-Z-6'-enecosamide) sphingosine, isovitexin, and $3 \beta-O$-D-glucopyranosyl-24 $\xi$-ethylcholest-5-ene have been identified and isolated from this plant species. The present review attempts to bridge the gap between traditional use and pharmacological studies of $M$. loriformis while improving their existing therapeutic agents and product applications based on this plant.
\end{abstract}

\section{Introduction}

Safety is one of the major concerns in the drug development. According to the Food and Drug Administration (FDA), to bring a new pharmaceutical drug to the market, the lead compounds in drug discovery will have to get through drug evaluation process that includes preclinical and clinical phases [1]. Plant kingdom serves as abundance and safe source for new drug discovery. The analysis reported from 1981 to 2019 by Newman and Cragg (2020) revealed that approximately $23.5 \%$ sources of new and approved drugs by FDA for the treatment of human diseases are from natural sources such as $11.5 \%$ from natural product mimics and $3.2 \%$ from total synthesis, but the pharmacophore is still from a natural product [2]. Thus, we suggest that the natural product continues to play a major role in the drug discovery and development process.
Plants survive in an environmental stressor with limitation on temperature, light, water, and nutrient availability by biosynthesizing their specific secondary metabolites. These metabolites enable plant growth, reproduction, and defense against environmental threats $[3,4]$. In this manner, a plant may possess various biological activities because of the secondary metabolites in individual plants. This could lead to their potential used as therapeutic agents with anticancer, antioxidant, anti-inflammatory, analgesic, antiaging, antiviral, antipyretic, and antimicrobial properties. Murdannia loriformis (Hassk.) Rolla Rao et Kammathy (M. loriformis) is among the popular folk medicines consumed by cancer and diabetes patients in Thailand $[5,6]$. This plant has gained much attention in Thailand after intake of $M$. loriformis juice. It is reputed for retarding cancer progression besides enabling symptomatic relief resulting from modern therapy $[7,8]$. M. loriformis is also one of the 
traditional Chinese remedies used to treat detoxification and respiratory tract complaints [8]. Owing to their therapeutic potential, several scientific reports have been documented to support the claims [7, 9-16]. This review provides a comprehensive summary of the updated information of ethnomedicinal use, phytochemistry, pharmacology, and toxicology of $M$. loriformis. Therefore, the present review may bridge the gap between traditional use and pharmacological studies of $M$. loriformis in addition to improving the existing therapeutic agents and product application of M. loriformis.

The literature was searched from different databases using electronic search engines: Google Scholar, Science Direct, PubMed, CAB Direct, Springerlink, MEDLINE Complete, SAGE Journals Online, Ovid LWW, Scopus, Wiley Online Library, EBSCOhost, JetP, thesis, recognized books, and conference proceedings up to March 2021. The keywords used were "Murdannia loriformis," "Beijing grass," and "Angel grass." The search was limited to articles published in Malay and English languages. The scientific name of Murdannia loriformis was validated using International Plant Name Index (http://www.ipni.org) and The Plant List (http://www.theplantlist.org) databases.

\section{Botany}

Murdannia loriformis (Hassk.) Rolla Rao et Kammathy (M. loriformis) belongs to the family Commelinaceae, and it originated from India [16]. The plant species is distributed in the forests and grassy slopes of Asia, particularly China and Thailand [17]. This plant is attracting attention in Malaysia for its medicinal potential such as psoriasis and eczema [18]. M. loriformis is conspecific to $M$. nudiflora because of their similarity in habit, inflorescence, and capsule. However, M. loriformis differs from $M$. nudiflora as follows: sheaths of cauline leaves ciliate only on 1 side of mouth, main stem undeveloped, seeds finely white, reticulate, neither pitted nor verrucose, and pedicels slightly curved. $M$. loriformis is known as "Beijing grass," or "Angel grass" in English, "Rumput Cina," "Rumput Beijing," and Rumput Siti Khatijah in Malay and "Ya Pak King" in Thai (Figure 1) $[12,18,19]$.

It is suggested in The Plant List [20] and Flora of China [17] that plants of Aneilema angustifolium, Aneilema loriforme, Aneilema terminale, Murdannia angustifolia, and Aneilema nudiflorum are synonymous with $M$. loriformis. Previously, the genus of Murdannia Royle was categorized under the genus of Aneilema, a large and heterogeneous group. However, after revision of Aneilema genus, Murdannia has been split off from the genus because of their differences in capsule, flower, and staminode structure [21].

Taxonomical of $M$. loriformis can be classified as follows:

\begin{tabular}{|c|c|}
\hline Scientific name & Murdannia loriformis (Hassk.) Rolla Rao et Kammathy \\
\hline Common name & $\begin{array}{l}\text { Beijing grass, Rumput Cina, Rumput Beijing, Rumput Siti Khatijah, Angel } \\
\text { grass, Ya Pak King }\end{array}$ \\
\hline Family & Commelinaceae \\
\hline Genus & Murdannia \\
\hline Species & M. loriformis \\
\hline
\end{tabular}

M. loriformis is a monocotyledonous and perennial herb. It is approximately $10 \mathrm{~cm}$ tall. The basal leaves of $M$. loriformis are simple, blades linear, alternate, and glabrous. The main stem is undeveloped; fertile stems are numerous, arising from the rosette, diffuse or ascending, measure $15-50 \mathrm{~cm}$, glabrous or puberulent on one side, extremely rarely densely hirsute. The basal leaves with a linear blades measure $5-15 \mathrm{~cm}$ long and 6-9 mm wide. The flowers are terminal, inflorescence and densely panicle. The involucral bracts are leaflike but smaller than leaves, distal ones extremely small, less than $10 \mathrm{~mm}$, and peduncle about $2.5 \mathrm{~cm}$. Pedicels slightly curved, translucent bracts about $4 \mathrm{~mm}$, sepal, ovate-elliptic, about $3 \mathrm{~mm}$, herbaceous. The petals are blue or bluish violet and obovate orbicular. The capsule is ovoid globose and trigonous, about $3-4 \mathrm{~mm}$. The seeds have two per valve: yellow-brown, radiate, striate, and finely white reticulate. The rhizomes are absent. The roots are fibrous that measures approximately $0.5-1 \mathrm{~mm}$ in diameter, glabrous or tomentose. The flower season is between May and October [8, 17].

\section{Ethnomedicinal Uses}

The plant $M$. loriformis is used traditionally for self-treatment by cancer patients among rural communities in Thailand. Different cancer patients have claimed that consuming fresh drinks of $M$. loriformis could prolong life and reduce the adverse effects of radiation and chemotherapy $[8,11]$. In Thai folklore medicine, $M$. loriformis has extensively been used as pain relief from bronchitis, diabetes treating, laxative, and cooling agent [5]. Sometimes, the whole plants are decoction with water and taken orally for hepatomegaly treatment [22]. The leaf parts of M. loriformis are often used to treat various ailments (Table 1 ). Their wide use has led to the formulation of many commercial products in the market based on $M$. loriformis, including cream [31], gel [32], capsule [33, 34], tablet [35], tea [36], gummy [37], and mask [38] for cosmetic, nutraceutical, and pharmaceutical purposes. 


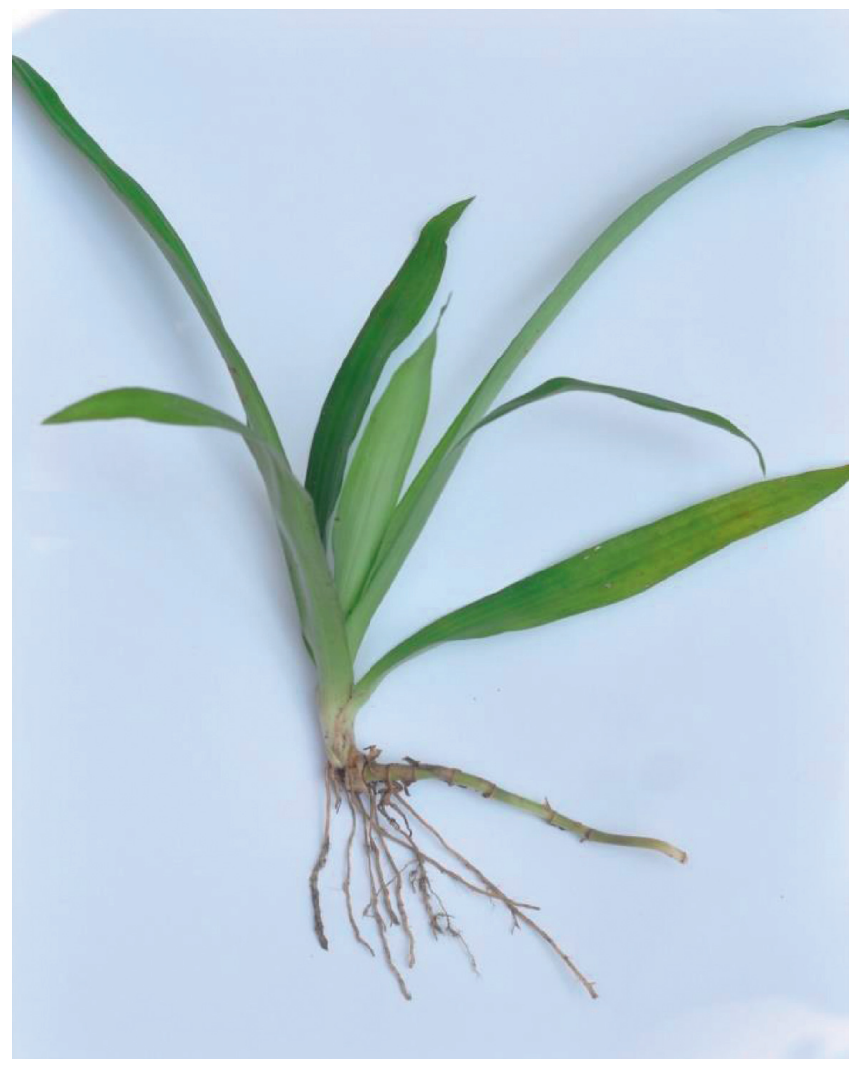

(a)

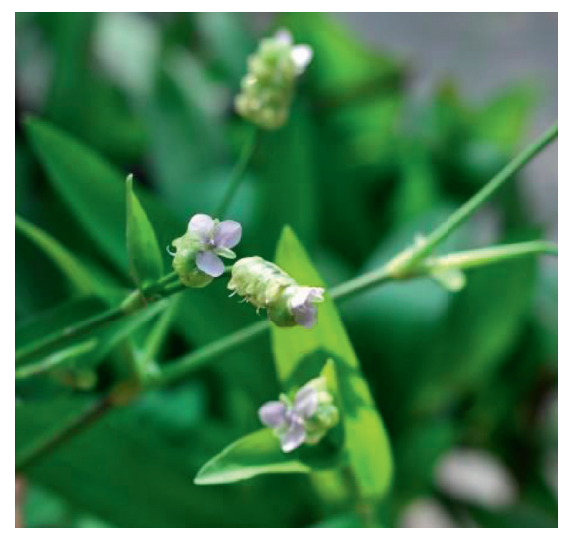

(b)

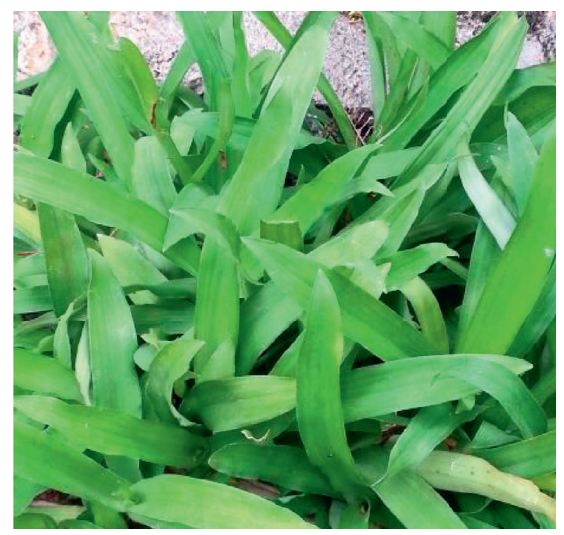

(c)

Figure 1: Murdannia loriformis (Hassk.): (a) whole plant including roots; (b) flowers; (c) leaves.

\section{Phytochemistry}

The phytochemical screening of $M$. loriformis revealed that it is has phenolics, flavonoids, condensed tannin [39], and plant membrane lipid [40] in this plant's extract. With extraction condition of $30 \mathrm{~min}$ at $70^{\circ}$, the antioxidant activity and total phenolic content of $M$. loriformis were $30.43 \mathrm{mg}$ $\mathrm{TE} / \mathrm{mL}$ and $25.52 \mathrm{mg} \mathrm{GAE} / \mathrm{mL}$, respectively [41]. Pongsathorn and co-workers quantified chlorophyll $a$ $(47.88 \pm 7.01 \mu \mathrm{g} / \mathrm{g} \mathrm{dw})$ and chlorophyll $b(50.78 \pm 3.89 \mu \mathrm{g} / \mathrm{g}$ $\mathrm{dw}$ ) in the methanolic extract of $M$. loriformis [42], but carotenoid pigment was not detected. Another phytochemical screening by Phattalung et al. detected phytoconstituents, namely, alkaloids, and steroids in the whole plant extract of 95\% ethanol $M$. loriformis [43]. Antioxidant activity of M. loriformis measured by DPPH radical scavenging activity was $91.5 \%$ at a concentration of $500 \mu \mathrm{g} / \mathrm{mL}$ [42].

Jiratchariyakul et al. successfully isolated phytosterol glucosides, glycosphingolipid (Figure 2(a)), and digalactosyl diglyceride from methanol fraction of ethanolic extract $M$. loriformis plant [7]. This fraction and their pure compound of glycosphingolipid, namely, $\beta$-O-D-glucopyranosyl- 
TABle 1: Ethnomedicinal uses of M. loriformis.

\begin{tabular}{|c|c|c|c|c|}
\hline Indications & Plant part used & Herbal preparation & Prescription and dosage form & Reference \\
\hline Cough, flu and allergies & Fresh leaves & NAD & NAD & [23] \\
\hline $\begin{array}{l}\text { Hemostatic and diuretic remedies, } \\
\text { antioxidant and anticarcinogen }\end{array}$ & $\begin{array}{l}\text { Fresh aerial } \\
\text { plant }\end{array}$ & Fresh juice & $\begin{array}{c}\text { Take orally, twice a day (Morning } \\
\text { and night) }\end{array}$ & [7] \\
\hline $\begin{array}{l}\text { Detoxification and respiratory } \\
\text { tract complaints }\end{array}$ & NAD & NAD & NAD & {$[11]$} \\
\hline Cooling effect, laxative & NAD & NAD & NAD & {$[5]$} \\
\hline $\begin{array}{l}\text { Pain relief from bronchitis, } \\
\text { antimutagenic }\end{array}$ & $\begin{array}{l}\text { Whole fresh } \\
\text { plants }\end{array}$ & Extraction with $80 \%$ ethanol & NAD & {$[16,24]$} \\
\hline $\begin{array}{l}\text { Diabetes mellitus, throat } \\
\text { infections, pneumonia and } \\
\text { inflammation (inflamed wound) }\end{array}$ & NAD & NAD & NAD & {$[11,12,25]$} \\
\hline Cures lymphadenopathy & NAD & NAD & NAD & {$[26]$} \\
\hline $\begin{array}{l}\text { Cancer treatment, } \\
\text { chemopreventive effects }\end{array}$ & NAD & NAD & NAD & {$[16,27,28]$} \\
\hline $\begin{array}{l}\text { Strengthen immune system, fever, } \\
\text { ulcer and to treat colon and breast } \\
\text { cancer }\end{array}$ & Fresh leaves & $\begin{array}{l}\text { Blend } 20-25 \text { of fresh leaves with } \\
\text { apple in } 1 \text { glass of water. Grind } \\
20-25 \text { of fresh leaves, filtered }\end{array}$ & $\begin{array}{l}\text { Take orally, } 1 \text { glass, } 2-3 \text { times a day or } \\
2 \text { tablespoon (concentrated) or } 30 \mathrm{ml} \\
\text { (diluted), } 2-3 \text { times a day }\end{array}$ & [29] \\
\hline Psoriasis and eczema & Fresh leaves & $\begin{array}{l}\text { Blend } 6 \text { of fresh leaves with } 4 \\
\text { tablespoons of water, filtered }\end{array}$ & $\begin{array}{l}\text { Take orally, twice a day (morning } \\
\text { and night) }\end{array}$ & {$[30]$} \\
\hline $\begin{array}{l}\text { Kidney stones relief and kidney } \\
\text { detox }\end{array}$ & $\begin{array}{l}\text { Whole fresh } \\
\text { plant (include } \\
\text { root) }\end{array}$ & $\begin{array}{l}\text { Decoction } 7-9 \text { of whole plants } \\
\text { with } 1.5 \mathrm{~mL} \text { water }\end{array}$ & (25) & [30] \\
\hline To tenderize meet & Fresh leaves & Tied in a knot, placed in cooking & NAD & [30] \\
\hline
\end{tabular}

NAD: Not appropriately described.

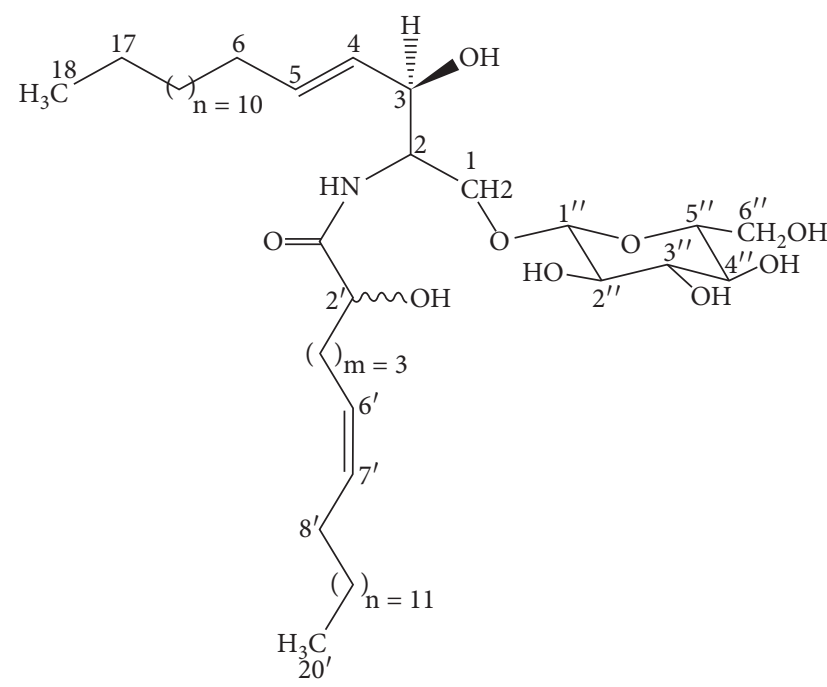

(a)<smiles></smiles>

(c)<smiles>COc1cc(C(=O)O)cc(OC)c1O</smiles>

(b)<smiles>N[C@@H](Cc1ccccc1)C(=O)O</smiles>

(d)

Figure 2: Continued. 


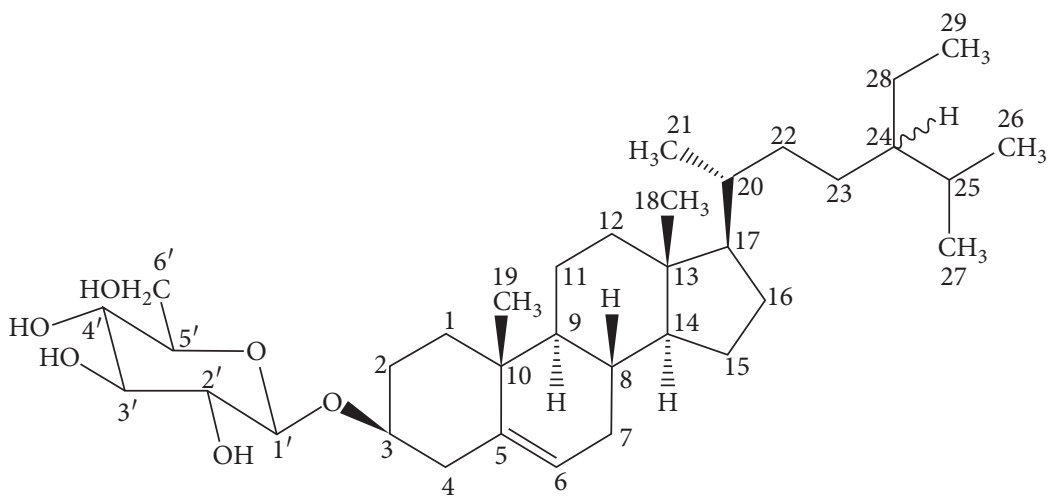

(e)

Figure 2: Phytochemicals identified and isolated from M. loriformis. (a) $\beta$-O-D-Glucopyranosyl-2-( $2^{\prime}$-hydroxy-Z- $6^{\prime}$-enecosamide).

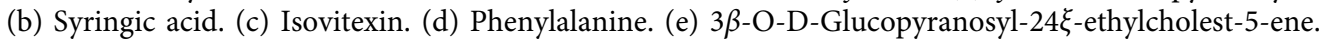

2-(2'-hydroxy-Z-6' -enecosamide) sphingosine have moderate cytotoxic activity against breast and colon cancer cell lines. Further isolation of water-methanol fraction from the same extract established the presence of chalconoid substances, syringic acid (Figure 2(b)), and isovitexin (Figure 2(c)). According to the study, the aqueous fractions of the ethanolic extract of $M$. loriformis have inorganic salt $(\mathrm{Na}, \mathrm{K}$, and $\mathrm{Mg}$ ) and phenylalanine (Figure 2(d)) [7]. Phenylalanine is a type of aromatic amino acids in the plant for protein synthesis besides serving as precursors for the biosynthesis of flavonoids through shikimate pathway resulting in the formation of phenolic compounds such as trans-Cinnamic acid, p-coumaric acid, condensed tannins, and anthocyanins. [44]. Also, a steroidal glucoside, namely, $3 \beta-O-D$-glucopyranosyl$24 \xi$-ethylcholest-5-ene (Figure $2(\mathrm{e})$ ) has been found in this plant, although these compounds may have no activity to cancer cell lines [11].

Pinitsoontorn et al. [26] quantified the contents of oxalate (89.50 ppm/g dry wt.) and calcium (54.67 ppm/g dry wt.) in $M$. loriformis leaves. However, because this plant is consumed freshly or as herbal tea, further research should focus on their toxicity level of oxalate content related to their safety concern because high levels of oxalate will risk to calcium oxalate kidney stone problem.

\section{Pharmacological Effects}

Several scientific reports support the traditional use of $M$. loriformis in treating different ailments $[7,10,12,13,16,26,42,45,46]$. Although some may not directly correlate with their traditional uses, they provide insight into its therapeutic potential and bioactive properties. The antioxidant, anticancer, antimutagenicity, antiinflammatory, antimicrobial, and immunomodulatory effects of M. loriformis extract are summarized in Table 2.

5.1. Antioxidant Activity. The earliest study on the antioxidant activity of this plant was reported by Pinitsoontorn and co-workers [26]. Antioxidant screening studies of over 20 different Thai herbal teas including $M$. loriformis revealed that the antioxidant activity of the tea and its phenolic content was among the average compared to other commercial teas. Pongsathorn [42] further studied the content of phenolics and pigments from methanol extract of $M$. loriformis to understand their antioxidant activity. The results revealed that the phenolic content of $M$. loriformis extract was less $<10 \mathrm{mg} \mathrm{GAE} / \mathrm{g}$ extract, and their chlorophyll $a$ and chlorophyll $b$ contents were 47.88 and $50.78 \mu \mathrm{g} / \mathrm{g}$ dry weight, respectively. The extract had non-carotenoids. Adisakwattana et al. reported phenolic content in aqueous extract of $M$. loriformis as $12.2 \mathrm{mg} \mathrm{GAE} / \mathrm{g}$ extract [51].

Techaratanakrai et al. further studied the antioxidant activity of $M$. Loriformis with a focus on the relation to temperature and diffusion time using DPPH assay method [41]. According to Techaratanakrai et al., the antioxidant activity of $M$. Loriformis demonstrated a contrasting effect on total phenolic content regarding infusion time and temperature. This study revealed that the phenolic groups have no significant effect on the antiradical activity measured by the DPPH radical scavenging activity assay; rather, the influence was contributed by other groups of antioxidant contributors such as chlorophyll. According to the authors, the optimum parameter suggested for the extraction condition was $70^{\circ} \mathrm{C}$ for 30 -minute infusion time to obtain the highest antioxidant activity of $30.43 \mathrm{mg} \mathrm{TE} / \mathrm{mL}$ and a total phenolic content of $25.52 \mathrm{mg}$ GAE/mL [41]. Kittipongpittaya et al. [12] studied the potential benefit from antioxidant biological activity of $M$. loriformis extract in food systems. The results demonstrated that $200-800 \mathrm{ppm}$ of M. loriformis extracts applied to pork lard and soybean oil reduced the rate of peroxide values in the oil dependently over 14 days of storage time.

5.2. Antimutagenicity and Anticancer Activity. The chemopreventive effects of $M$. loriformis were initially demonstrated by Rearungchom in 1993 [46] with a short-term antimutagenic effect of methanolic extract of $M$. loriformis on aflatoxin $\mathrm{B} 1\left(\mathrm{AFB}_{1}\right)$ mutagenesis. During preincubation technique using Salmonella typhimurium TA 98 and TA 100 with and without metabolic activation, the tested extract did 
TABle 2: Pharmacological studies on $M$. loriformis.

\begin{tabular}{|c|c|c|c|c|c|c|}
\hline Activity tested & $\begin{array}{l}\text { Plant } \\
\text { part }\end{array}$ & Extract & $\begin{array}{l}\text { Experimental } \\
\text { procedures }\end{array}$ & $\begin{array}{c}\text { Dosage } \\
\text { concentration }\end{array}$ & Results & References \\
\hline \multirow{9}{*}{ Antioxidant } & $\begin{array}{l}\text { Whole } \\
\text { plant }\end{array}$ & $80 \%$ ethanol & $\begin{array}{l}\text { 1,1-Diphenyl-2- } \\
\text { picrylhydrazyl (DPPH) } \\
\text { assay. }\end{array}$ & $200-800 \mathrm{ppm}$ & $\begin{array}{l}\text { Showed a concentration- } \\
\text { dependent DPPH radical } \\
\text { scavenging activity. }\end{array}$ & {$[12]$} \\
\hline & & & $\begin{array}{l}\text { Determination of } \\
\text { peroxide values } \\
\text { according to AOCS } \\
\text { method. }\end{array}$ & $0-800 \mathrm{ppm}$ & $\begin{array}{l}\text { Rate of peroxide values in } \\
\text { pork lard and soybean oil } \\
\text { containing } M \text {. loriformis have } \\
\text { been reduced. }\end{array}$ & {$[12]$} \\
\hline & Leaves & $\begin{array}{l}\text { Deionized } \\
\text { water }\end{array}$ & $\begin{array}{l}\text { Ferric reducing } \\
\text { antioxidant power } \\
\text { (FRAP) assay. }\end{array}$ & NAD & $\begin{array}{l}\text { Antioxidant activity and total } \\
\text { phenolic content of } \\
\text { M. loriformis extract was } \\
4.14 \mathrm{mM} \mathrm{Fe} \mathrm{(II)/g} \mathrm{dry} \mathrm{wt} \mathrm{and} \\
46.96 \mathrm{mg} \mathrm{GAE} / \mathrm{g} \text { dry wt, } \\
\text { respectively. }\end{array}$ & {$[26]$} \\
\hline & & & $\begin{array}{l}\text { Determination of total } \\
\text { phenolic content using } \\
\text { Folin-Ciocalteu } \\
\text { method. }\end{array}$ & NAD & $\begin{array}{l}\text { Increased temperature of } \\
\text { extraction extremely } \\
\text { decreased the DPPH radical } \\
\text { scavenging activity. }\end{array}$ & \\
\hline & & & Investigation the effect & & & \\
\hline & NAD & Water & $\begin{array}{l}\text { of time and } \\
\text { temperature on } \\
\text { antioxidant activity of } \\
\text { M. loriformis by DPPH } \\
\text { assay. }\end{array}$ & NAD & $\begin{array}{l}\text { Increased time of extraction } \\
\text { slightly increased the total } \\
\text { phenolic content of the } \\
\text { extract. }\end{array}$ & {$[41]$} \\
\hline & & & $\begin{array}{l}\text { Folin-Ciocalteu } \\
\text { method. }\end{array}$ & NAD & $\begin{array}{l}\text { Total phenolic content of the } \\
\text { extract was less }<10 \mathrm{mg} \text { GAE/ } \\
\text { g extract. }\end{array}$ & \\
\hline & & & $\begin{array}{l}\text { Folin-Ciocalteu } \\
\text { method. }\end{array}$ & $\begin{array}{c}0.05-500 \mu \mathrm{g} / \\
\mathrm{mL}\end{array}$ & $\begin{array}{l}\text { Exhibited a concentration- } \\
\text { dependent DPPH radical } \\
\text { scavenging activity. }\end{array}$ & \\
\hline & NAD & $\begin{array}{l}95 \% \\
\text { methanol }\end{array}$ & $\begin{array}{l}\text { DPPH assay. } \\
\text { Determination of } \\
\text { pigment content } \\
\text { according to } \\
\text { Lichtenthaler and } \\
\text { Wellburn [47]. } \\
\end{array}$ & $1000 \mu \mathrm{g} / \mathrm{mL}$ & $\begin{array}{c}\text { Chlorophyll } a \text { and } \\
\text { chlorophyll } b \text { were } \\
\text { quantified in } M \text {. loriformis } \\
\text { extract. }\end{array}$ & {$[42]$} \\
\hline \multirow{3}{*}{ Antimutagenicity } & $\begin{array}{l}\text { Whole } \\
\text { plant }\end{array}$ & $80 \%$ ethanol & $\begin{array}{c}\text { Salmonella mutation } \\
\text { assay }\end{array}$ & $\begin{array}{l}0.1-1.0 \mathrm{~g} / \mathrm{kg} \\
\text { body weight }\end{array}$ & \multirow{3}{*}{$\begin{array}{c}\text { M. loriformis showed } \\
\text { antimutagenicity activity by } \\
\text { its inhibitory effects on } \\
\text { azoxymethane-induced } \\
\text { DNA methylation and } \\
\text { aberrant crypt focus } \\
\text { formation in male F344 rats. } \\
\text { Methanol extract showed } \\
\text { antimutagenicity activity } \\
\text { against aflatoxin } \mathrm{B}_{1}\left(\mathrm{AFB}_{1}\right) \\
\text { mutagenesis in the short } \\
\text { term. } \\
\text { Multiple doses of treatments } \\
\text { have decreased AF-albumin } \\
\text { adduct levels. }\end{array}$} & {$[10,15]$} \\
\hline & $\begin{array}{l}\text { Whole } \\
\text { plant }\end{array}$ & Methanol & $\begin{array}{c}\text { Salmonella mutation } \\
\text { assay. }\end{array}$ & $0.05 \mu \mathrm{g}$ & & {$[46]$} \\
\hline & $\begin{array}{l}\text { Whole } \\
\text { plant }\end{array}$ & $80 \%$ ethanol & $\begin{array}{c}\text { Competitive enzyme- } \\
\text { linked immuno- } \\
\text { sorbent assay (ELISA). }\end{array}$ & $\begin{array}{l}3 \mathrm{~g} / \mathrm{kg} \text { body } \\
\text { weight }\end{array}$ & & {$[16]$} \\
\hline
\end{tabular}


TABle 2: Continued.

\begin{tabular}{|c|c|c|c|c|c|c|}
\hline Activity tested & $\begin{array}{c}\text { Plant } \\
\text { part }\end{array}$ & Extract & $\begin{array}{c}\text { Experimental } \\
\text { procedures }\end{array}$ & $\begin{array}{c}\text { Dosage } \\
\text { concentration }\end{array}$ & Results & References \\
\hline \multirow[b]{2}{*}{ Anticancer } & $\begin{array}{l}\text { Whole } \\
\text { plant }\end{array}$ & $80 \%$ ethanol & $\begin{array}{c}\text { MTT assay. } \\
\text { Cytotoxicity of } \\
\text { M. loriformis was } \\
\text { determined against } \\
\text { breast (MCF7) and } \\
\text { colon (HT29) cancer } \\
\text { cell lines }\end{array}$ & $20-100 \mu \mathrm{g} / \mathrm{mL}$ & $\begin{array}{l}\text { Moderate cytotoxic activity } \\
\mathrm{ED}_{50} \text { less than } 10 \mu \mathrm{g} / \mathrm{mL} \text {. }\end{array}$ & [7] \\
\hline & $\begin{array}{l}\text { Whole } \\
\text { plant }\end{array}$ & $95 \%$ ethanol & $\begin{array}{c}\text { Cytotoxicity of } \\
\text { M. loriformis was } \\
\text { examined against } \\
\text { immortalized human } \\
\text { keratinocytes (HaCaT), } \\
\text { oral epithelial (HN4) } \\
\text { and carcinoma cell lines } \\
\text { of head and neck } \\
\text { squamous (HN12), } \\
\text { breast (MCF7) and } \\
\text { colon (HT29). }\end{array}$ & $\begin{array}{l}0-10.0 \mathrm{mg} / \\
\text { plate }\end{array}$ & $\begin{array}{c}\text { Showed antiproliferative } \\
\text { effect on HT29 and MCF7 } \\
\text { cells. }\end{array}$ & {$[45]$} \\
\hline Anti-inflammatory & $\begin{array}{c}\text { Aerial } \\
\text { part }\end{array}$ & $80 \%$ ethanol & $\begin{array}{c}\text { Carrageenan- and } \\
\text { arachidonic acid (AA)- } \\
\text { induced paw edema in } \\
\text { rat's assay. }\end{array}$ & $\begin{array}{l}100-400 \mathrm{mg} / \\
\mathrm{kg} \\
100-400 \mathrm{mg} / \\
\mathrm{kg}\end{array}$ & $\begin{array}{l}\text { M. loriformis extract } \\
\text { significantly reduced the } \\
\text { carrageenan-induced edema } \\
\text { formation of the rat paw. } \\
\text { Inhibited of AA-induced paw } \\
\text { edema in dose-dependent } \\
\text { manner. } \\
\text { M. loriformis extract } \\
\text { significantly lowered the } \\
\text { transudative weight. The } \\
\text { inhibitory effect of } \\
\text { granuloma formation was } \\
\text { well correlated with their } \\
\text { transudative weight. } \\
\text { M. loriformis did not affect } \\
\text { the gastric mucosa of rats } \\
\text { (ulcer index }=0 \text { ). }\end{array}$ & {$[13,48]$} \\
\hline \multirow{3}{*}{ Immunomodulatory effect } & \multirow[t]{2}{*}{ NAD } & $\begin{array}{c}\text { Pressed and } \\
\text { added of } \\
50-100 \mathrm{~mL} \\
\text { water. (Herb } \\
\text { juice) }\end{array}$ & $\begin{array}{l}\text { In vitro cellular } \\
\text { immunological assays. }\end{array}$ & $\begin{array}{c}500 \mu \mathrm{g} / \mathrm{mL} \\
\text { (herbal juice) }\end{array}$ & $\begin{array}{c}\text { Herb juice and isolated } \\
\text { compound namely } \\
\text { glycosphingolipid } \beta-O-D- \\
\text { glucopyranosyl-2-(2'- } \\
\text { hydroxy-Z- } 6^{\prime}-\text {-enecosamide) } \\
\text { sphingosine have increased } \\
\text { PBMC proliferation in the } \\
\text { presence of the mitogen PHA } \\
\text { (phytohemagglutinin). }\end{array}$ & \multirow[b]{3}{*}[24]{} \\
\hline & & $\begin{array}{l}\text { Ethanol } \\
\text { (isolated } \\
\text { compound) }\end{array}$ & & $\begin{array}{l}0.01 \mu \mathrm{g} / \mathrm{mL} \\
\text { (isolated } \\
\text { compound) }\end{array}$ & $\begin{array}{c}\text { Both increase the expression } \\
\text { of CD 3,4:CD 3,8 ratio in } \mathrm{T} \\
\text { lymphocytes. }\end{array}$ & \\
\hline & $\begin{array}{l}\text { Whole } \\
\text { plant }\end{array}$ & $80 \%$ ethanol & $\begin{array}{l}\text { Lymphocyte activation } \\
\text { assay. }\end{array}$ & $1-200 \mu \mathrm{g} / \mathrm{mL}$ & $\begin{array}{l}\text { Decrease of T- and B-cell } \\
\text { proliferation with the } \\
\text { presence and absence of } \\
\text { mitogen. } \\
\text { The water extract } \\
\text { significantly decreased PHA } \\
\text { and pokeweed mitogen } \\
\text { (PWM)-induced lymphocyte } \\
\text { proliferation. }\end{array}$ & \\
\hline
\end{tabular}


TABle 2: Continued.

\begin{tabular}{|c|c|c|c|c|c|c|}
\hline Activity tested & $\begin{array}{c}\text { Plant } \\
\text { part }\end{array}$ & Extract & $\begin{array}{c}\text { Experimental } \\
\text { procedures }\end{array}$ & $\begin{array}{c}\text { Dosage } \\
\text { concentration }\end{array}$ & Results & References \\
\hline Antipyretic & $\begin{array}{c}\text { Aerial } \\
\text { part }\end{array}$ & $80 \%$ ethanol & $\begin{array}{c}\text { Yeast-induced } \\
\text { hyperthermia assay }\end{array}$ & $400 \mathrm{mg} / \mathrm{kg}$ & $\begin{array}{c}\text { Reduced the rectal } \\
\text { temperature to normal in } \\
30 \text { min and lasted for } \\
180 \text { min. }\end{array}$ & [13] \\
\hline Analgesic & $\begin{array}{c}\text { Aerial } \\
\text { part }\end{array}$ & $80 \%$ ethanol & Formalin test & $20-80 \mathrm{mg} / \mathrm{kg}$ & $\begin{array}{l}\text { Reduced the licking time in } \\
\text { early and late phases. }\end{array}$ & [12] \\
\hline \multirow[b]{2}{*}{ Antimicrobial } & $\begin{array}{l}\text { Whole } \\
\text { plant }\end{array}$ & NAD & $\begin{array}{l}\text { Disc diffusion and } \\
\text { broth dilution methods. }\end{array}$ & $1.25 \mathrm{mg} / \mathrm{mL}$ & $\begin{array}{l}\text { Have no activity against } \\
\text { Propionibacterium acnes. } \\
\text { Inhibited the growth of } \\
\text { Staphylococcus epidermidis at } \\
\text { MIC value of } 1.25 \mathrm{mg} / \mathrm{mL} \text {. } \\
\text { However, the MBC value was } \\
>5 \mathrm{mg} / \mathrm{mL} \text {. }\end{array}$ & [9] \\
\hline & $\begin{array}{l}\text { Whole } \\
\text { plant }\end{array}$ & $95 \%$ ethanol & $\begin{array}{c}\text { Antibacterial and } \\
\text { antifungal assays. } \\
\text { M. loriformis extract } \\
\text { was tested for light- } \\
\text { mediated activities } \\
\text { against Bacillus subtilis, } \\
\text { Staphylococcus aureus } \\
\text { K147 methicillin- } \\
\text { sensitive (Ms), } \\
\text { Escherichia coli DC10, } \\
\text { E. coli (wild), } \\
\text { Pseudomonas } \\
\text { aeruginosa } 187 \text { (wild), } \\
\text { Candida albicans and } \\
\text { Aspergillus fumigatus. }\end{array}$ & $1 \mathrm{mg} / \mathrm{mL}$ & $\begin{array}{l}\text { Inhibited the growth of } \\
\text { B. subtilis under the influence } \\
\text { of UV light with zone } \\
\text { inhibition diameter of } \\
8-12 \mathrm{~mm} \text {. }\end{array}$ & {$[23]$} \\
\hline Antibacterial & $\begin{array}{l}\text { Whole } \\
\text { plant }\end{array}$ & Ethanol & $\begin{array}{c}\text { Disc diffusion method. } \\
\text { M. loriformis was } \\
\text { investigated against } \\
\text { S. aureus }\end{array}$ & $\begin{array}{c}0.5-5000 \mu \mathrm{g} / \\
\mathrm{mL}\end{array}$ & No antibacterial activity. & {$[43,49,50]$} \\
\hline Gastroprotective activity & $\begin{array}{l}\text { Whole } \\
\text { plant }\end{array}$ & $80 \%$ ethanol & $\begin{array}{c}\text { Gastroprotective } \\
\text { activity studies using } \\
\text { three different models: } \\
\text { EtOH/HCl, } \\
\text { indomethacin, and } \\
\text { restraint water } \\
\text { immersion stress. } \\
\text { Gastric visible mucus } \\
\text { secretion. }\end{array}$ & $\begin{array}{l}100-400 \mathrm{mg} / \\
\mathrm{kg}\end{array}$ & $\begin{array}{c}\text { M. loriformis extract } \\
\text { significantly inhibited gastric } \\
\text { ulcer formation induced by } \\
\text { EtOH/HCl, indomethacin } \\
\text { and stress. } \\
\text { Significantly increased the } \\
\text { amount of gastric wall } \\
\text { mucus. } \\
\text { Reduced gastric acid } \\
\text { secretion in the pylorus } \\
\text { ligation model. }\end{array}$ & [14] \\
\hline
\end{tabular}


TABLE 2: Continued.

\begin{tabular}{|c|c|c|c|c|c|c|}
\hline Activity tested & $\begin{array}{c}\text { Plant } \\
\text { part }\end{array}$ & Extract & $\begin{array}{c}\text { Experimental } \\
\text { procedures }\end{array}$ & $\begin{array}{c}\text { Dosage } \\
\text { concentration }\end{array}$ & Results & References \\
\hline $\begin{array}{l}\text { Inhibition of pancreatic } \\
\text { lipase and pancreatic } \\
\text { cholesterol esterase, } \\
\text { cholesterol micelle } \\
\text { formation and bile acid } \\
\text { binding. }\end{array}$ & $\begin{array}{l}\text { Whole } \\
\text { plant }\end{array}$ & Water & $\begin{array}{l}\text { Pancreatic lipase } \\
\text { inhibition assay. } \\
\text { Pancreatic cholesterol } \\
\text { esterase inhibition } \\
\text { assay. } \\
\text { Cholesterol } \\
\text { micellization assay. }\end{array}$ & NAD & $\begin{array}{c}\text { Inhibited pancreatic lipase } \\
\text { activity in dose-dependent } \\
\text { manner } \\
\left(\mathrm{IC}_{50}=0.11 \pm 0.01 \mathrm{mg} / \mathrm{mL}\right) \\
\text { Inhibited pancreatic } \\
\text { cholesterol esterase activity } \\
\text { about } 10-22 \% \\
\left(\left(\mathrm{IC}_{50}=>3 \mathrm{mg} / \mathrm{mL}\right) .\right. \\
\text { Moderated cholesterol } \\
\text { micellization inhibition } \\
(18.01 \pm 1.44 \%) \\
\text { M. loriformis extract has bind } \\
\text { to glycodeoxycholic acid, } \\
\text { taurocholic acid and } \\
\text { taurodeoxycholic acid at } \\
30.31 \pm 6.88 \%, 28.70 \pm 2.08 \% \\
\text { and } 6.52 \pm 0.88 \%, \\
\text { respectively. }\end{array}$ & {$[51]$} \\
\hline $\begin{array}{l}\text { Inhibition of } \\
\alpha \text {-glucosidase, pancreatic } \\
\alpha \text {-amylase and protein } \\
\text { glycation activities. }\end{array}$ & $\begin{array}{l}\text { Whole } \\
\text { plant }\end{array}$ & Water & $\begin{array}{l}\text { Phytochemical analysis. } \\
\text { Intestinal } \alpha \text {-glucosidase } \\
\quad \text { inhibitory assay. }\end{array}$ & $1 \mathrm{mg} / \mathrm{mL}$ & 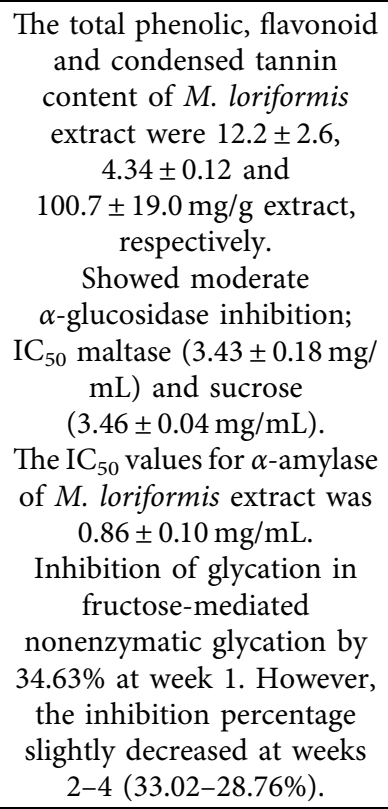 & [39] \\
\hline
\end{tabular}

not demonstrate any mutagenic activity towards $\mathrm{AFB}_{1}$ but demonstrated inhibitory effect towards the respective carcinogen $\left(\mathrm{AFB}_{1}\right)$.

Further in vivo studies were conducted by Vinitketkumnuen et al. to determine the levels of serum aflatoxin-albumin (AF-albumin) adduct formation in rats after exposure to $\mathrm{AFB}_{1}[16]$. $\mathrm{AFB}_{1}$ is a potent fungal toxin that initiates carcinogenesis in human and animal liver [52]. In this chemopreventive study, the rats treated with $3 \mathrm{~g} / \mathrm{kg}$ body weight (bw) of $M$. loriformis extract were treated with single and multiple doses of $250 \mu \mathrm{g} / \mathrm{kg}$ bw $\mathrm{AFB}_{1}$. The results suggest the ability of $M$. loriformis extract to influence the formation of AF-albumin adduct, whereas the rats treated with a single dose of $M$. loriformis extract appeared to modulate the rate of AF-albumin adduct formation. However, multiple doses of the treatment demonstrated slight decrease in the
AF-albumin adduct levels. This was probably due to longterm intervention with $M$. loriformis extract through $\mathrm{AFB}_{1}$ exposure. This might have caused formation of enzymes associated with carcinogen detoxification, leading to diminishing rates of $\mathrm{AFB}_{1}$ formation [53].

An extended chemopreventive study by Intiyot et al. described the antimutagenic effects of the extract towards mutagens and their inhibitory effects on azoxymethaneinduced DNA methylation and aberrant crypt focus (ACF) formation in male rats [10]. From the results, M. loriformis extract demonstrated antimutagenic activity against the tested heterocyclic amines in dose-dependent manner and decreased the number of ACF (with more than 3 aberrant crypts per focus) formation. ACF with more than three crypts per focus was reported to be associated with precolorectal cancer development $[10,54]$. Thus, treatment with 
M. loriformis extract could prevent azoxymethane-induced aberrant crypt foci by acting as a suppressing or blocking agent in the progression of carcinogenesis.

$M$. loriformis extract had been reported to induce DTdiaphorase (DTD) activity when tested on a murine hepatoma cell line (Hepa 1c1c7) [55]. DTD is an anticarcinogenic enzyme that acts as bioreductive agent for protecting tissues against carcinogens, mutagens, and cytotoxics. DTD in animals and humans tissues prevents free radicals formation [56]. In agreement with anticancer, studies by Koontongkaew and co-workers (2009) demonstrated the antiproliferative effectiveness of $M$. loriformis extract against colon and human breast cancer cell lines. Isolated compound of $M$. loriformis extract, namely, glycosphingolipid $\beta$-O-D-glucopyranosyl-2-( $2^{\prime}$-hydroxy-Z- $6^{\prime}$-enecosamide) sphingosine, has cytotoxic properties at concentrations of 8 , $14.5,12$, and $25 \mu \mathrm{g} / \mathrm{mL}$ against human breast, colon, lung, and liver cancer cell lines $[7,11]$. Hence, cytotoxic effects of $M$. loriformis discussed earlier scientifically supported the used of the extract in herbal tea consumption for cancer treatment and detoxification purposes practiced by folk medicine.

Moreover, the phenolic compounds such as isovitexin and syringic acid in the extract of $M$. loriformis may also attribute to their chemopreventive and anticancer effects $[57,58]$. According to Leon-Gonzalez et al. [58], oxidative stress increases proteins, lipids, and DNA damage in mice to induced colon carcinogenesis. Oxidative stress results in excessive levels of free radicals in humans. Plants with phenolic compounds or polyphenols have been recognized to have high levels of antioxidant activity and marked effects in the prevention of oxidative stresses $[59,60]$. Interestingly, in addition to their beneficial effects on antioxidant properties, there are significant evidences to suggest that prooxidant features of polyphenols contribute to their tumoricidal effects [61]. This is related to their ability to alter cellular redox status, whereas, in healthy cells, they act as antioxidant agents to prevent carcinogenesis, but, in cancer cells, they serve as prooxidants to destroy cancer cells [58].

5.3. Anti-Inflammatory Activity. Cumulative evidence suggests that inflammation may play a significant role in the development of precancerous lesions; hence, chronic inflammation is reported to increase risk for many cancers [62]. In other words, plant anticancer property may also exhibit anti-inflammatory activity, because of the associated factors of chronic inflammation such as disruption of DNA repair pathways, immunosuppression, and tissue destruction contribute to progression of carcinogenesis [63].

M. loriformis has been traditionally used to treat wound [11]. The scientific investigation of $M$. loriformis anti-inflammatory activity has been extensively studied by Kunnaja and co-workers [13]. The authors used carrageenan and arachidonic acid-induced hind paw edema models and cotton pellet-induced granuloma formation and transudation in rats for the acute and chronic inflammatory model, respectively. The finding demonstrates antiedematous effect of the extract in the tested assay through the inhibition effect of the extract towards paw edema in a concentration-dependent manner. M. loriformis extract responds to inflammation of rat paw in biphasic events. In the first few hours after injection, the extract suppressed histamine, kinins, and serotonin release. At the second phase, approximately 2.5-6h later, acute inflammation occurred through the release of prostaglandins by cyclooxygenase (COX) pathway $[13,64]$. It is hypothesized that treatment with $M$. loriformis extract may inhibit prostaglandin synthesis and block the cyclooxygenase pathway, whereas arachidonic acid can prevent the release of the two types of proinflammatory mediators, namely, prostaglandins and leukotrienes through dual inhibitors of COX and lipoxygenase (LOX) [65]. A comparative study of $M$. loriformis crude ethanolic extract and its fractions (hexane, chloroform, and ethyl acetate) have demonstrated that hexane fractions possesses stronger effects on nitric oxide reduction such as standard (L-NAME and indomethacin), and it is capable of reducing COX-2 protein expression in LPS-stimulated RAW264.7 cells [66]. Thus, we suggest that the active ingredient of anti-inflammatory of $M$. loriformis is the nonpolar compound [66].

Furthermore, studies on chronic inflammation assay using cotton pellet-induced granuloma formation and transudation in rats suggest that the extract of $M$. loriformis at a concentration of $400 \mathrm{mg} / \mathrm{kg}$ has the ability to control the alkaline phosphatase level in serum to a normal level. The inflammatory response of treated rats in the assay involving three phases, transudative, exudative, and proliferative phase resulted in decreased granuloma formation and transudation. Granuloma is a typical feature occurring in the early stage of chronic inflammatory process [67]. Thus, the inhibition effects of $M$. loriformis extract towards granuloma formation and transudation in rats suggest their ability to prevent the proliferative phase of chronic inflammation. Moreover, the extract demonstrated no significant effect on the gastric mucosa of rats when compared to control, indicating that the plant extract possesses desirable anti-inflammatory properties without ulcerogenic effect [14].

5.4. Immunomodulatory Effect. In principle, inflammation is a part of the immune response by preventing infection through production of proinflammatory cytokines and formation of inflammatory mediators, while anti-inflammatory activity of $M$. loriformis extract may attribute to its immunomodulatory properties because of the potential activation of immune effector in peripheral blood mononuclear cells (PBMC) [11]. In supporting one of its folklore uses for strengthening immune system [29], the literature is replete with evidence that $M$. loriformis extract improves immune system. Herb juice of $M$. loriformis extract using water contains glycosphingolipid $\beta$-O-D-glucopyranosyl-2( $2^{\prime}$-hydroxy-Z-6'-enecosamide) sphingosine. These compounds are reputed for increasing PBMC proliferation. This suggests the potential mitogenic properties of the extract [11]. In a comparative study, the researchers also reported that pure compound of glycosphingolipid was more effective than herb juice extract in stimulating PBMC proliferation. 
Both increase the expression of CD 3,4 molecules in $\mathrm{T}$ lymphocytes. However, in contrast to what has been reported by Punturee et al. [24], the ethanolic and water extract of $M$. loriformis at higher concentrations decreased T- and B-cell proliferation, which may be toxic to human PBMCs. Thus, the toxicity effects of $M$. loriformis extract on nonspecific cellular immune responses warrant further investigation.

5.5. Antipyretic Activity. Scientific reports justify the ethnomedicinal use of $M$. loriformis for fever treatment because of its antipyretic effect [13]. In this study, rats receiving M. loriformis extract at $400 \mathrm{mg} / \mathrm{kg}$ had their rectal temperature reduced to normal in $30 \mathrm{~min}$ and the effect persisted for $180 \mathrm{~min}$.

5.6. Analgesic Activity. In another study, Kunnaja et al. reported the potential analgesic effect of $M$. loriformis extract in dose-dependent manner to reduce paw licking time of the rats at both phases of formalin test [13]. The test involved two distinct phases, early (neurogenic) and late (inflammatory) phases, and it was carried out to check the effects of the extract on inflammatory pain. Analgesic action of the extract to reduce paw licking time in both phases suggested the extract property as an opioid drugs to produce morphine-like effect for pain relief [68]. Moreover, several researchers had reported the contribution of glycosides, alkaloids, tannins, and flavonoids contained in plants for analgesic action [68].

5.7. Antimicrobial and Antibacterial Activity. The antimicrobial activity of $M$. loriformis extract was evaluated against Gram-positive bacteria Propionibacterium acne (P. acnes) and Staphylococcus epidermidis (S. epidermidis) [9]. In the study, no bactericidal effect was observed against $P$. acnes; however, the tested extract inhibited the growth of $S$. epidermidis at a minimal inhibitory concentration (MIC) of $1.25 \mathrm{mg} / \mathrm{mL}$ [9]. In contrast, Kaewkod et al. [69] reported no antibacterial activity of ethanolic and aqueous extract of $M$. loriformis against $S$. epidermidis. The tested extracts of $M$. loriformis had no antibacterial activity against the other five bacteria causing skin diseases, namely, S. aureus, Bacillus sp., Ps. Aeruginosa, P. acnes, and MRSA. However, the ethanolic extract of $M$. loriformis exhibited antibacterial activity against $M$. luteus within the diameter of inhibition zone $7 \mathrm{~mm}$. MIC and minimal bactericidal concentrations (MBC) of ethanolic extract of $M$. loriformis against $M$. luteus were 62.5 and $125 \mathrm{mg} / \mathrm{mL}$, respectively. Meanwhile, aqueous extract of $M$. loriformis inhibited the growth of Corynebacterium sp. with the diameter of inhibition zone $20.0 \mathrm{~mm}$. MIC and MBC values of aqueous extract against bacterial strain of Corynebacterium sp. were 62.5 and $250 \mathrm{mg} / \mathrm{mL}$, respectively [69]. Both results indicate mild bactericidal activity of $M$. loriformis.

The potential using $M$. loriformis has been tested for light-activated antimicrobial activity against several bacterial and fungi. The ethanolic extract of $M$. loriformis under UV light produced a good inhibition zone $(8 \mathrm{~mm}$ to $12 \mathrm{~mm})$ against Bacillus subtilis, whereas no activity was observed under dark condition [23]. Besides, the extract demonstrated no activity in both conditions against Staphylococcus aureus, Escherichia coli, Pseudomonas aeruginosa, Candida albicans, and Aspergillus fumigatus. Similar findings by Phatthalung et al. [43], Limsuwan et al. [49], Chomnawang et al. [50], and Kaewkod et al. [69] have reported that M. loriformis extract was not effective against Staphylococcus aureus. This lightmediated activity of $M$. loriformis extract can be used in the future in the development of photochemistry and photochemotherapy approaches of this plant.

5.8. Gastroprotective Activity. The folklore use of M. loriformis to relieve inflammation $[11,12]$ is scientifically supported by their antigastric ulcer activity using three different models: ethanol/hydrochloric acid, indomethacin, and restraint water immersion stress [14]. M. loriformis extract in a dose-dependent manner significantly inhibited gastric ulcer formation induced by all tested models [14]. Oral administration of $M$. loriformis extract at $400 \mathrm{mg} / \mathrm{kg}$ significantly increased the amount of gastric wall mucus and reduced gastric acid secretion in the pylorus ligation model. In the model, pylorus ligature rats treated with $M$. loriformis extract had significantly reduced total acidity and gastric volume besides increasing gastric $\mathrm{pH}$ compared to the control group. Gastric ulcers occur because of imbalance between mucosal defensive mechanisms and endogenous or exogenous aggressive factors [70]. From the findings, M. loriformis demonstrates gastroprotective action on ulcer models through reduction of aggressive factor (gastric acid) and increasing of defensive mechanism (mucus). Thus, the growth of gastric wall mucus plays an important role as a defensive factor against gastrointestinal damage.

5.9. Other Activities. M. loriformis extract inhibits pancreatic lipase activity in dose-dependent manner $\left(\mathrm{IC}_{50}=0.11 \pm 0.01 \mathrm{mg} / \mathrm{mL}\right)$, albeit being less potent than the standard orlistat sample $\left(\mathrm{IC}_{50}=1.34 \pm 0.13 \mathrm{mg} / \mathrm{mL}\right)$ [51]. From the experiment, $M$. loriformis extract at $2 \mathrm{mg} / \mathrm{mL}$ inhibited pancreatic cholesterol esterase activity by $10 \%$ to $22 \%\left(\mathrm{IC}_{50}>3 \mathrm{mg} / \mathrm{mL}\right)$. Nonetheless, the tested extract was less potent compared with control, simvastatin $\left(\mathrm{IC}_{50}=0.08 \pm 0.01 \mathrm{mg} / \mathrm{mL}\right)$ [51]. In the in vitro cholesterol solubilization assay using artificial micelles, M. loriformis extract moderately inhibited cholesterol micellization by $18 \%$. Furthermore, the extract binds glycodeoxycholic acid and taurocholic acid by $30 \%$ and $28 \%$, respectively. However, taurodeoxycholic acid binds the extract by $6 \%$ [51]. The reason for using $M$. loriformis extract for antihyperlipidemic activity is based on delaying lipid digestion and absorption through gastrointestinal mechanisms. The mechanisms involve inhibition of pancreatic lipase, pancreatic cholesterol esterase activity, inhibition of cholesterol micellization, and bile acid binding [51]. Several studies had suggested that plant polyphenols have lipid lowering potential [71, 72]. Daily intake of polyphenol-enriched edible plants such as M. loriformis extract may delay the increase of postprandial 
hypertriacylglycerolemia and hypercholesterolaemia in obese individuals. Hence, the extract has been identified as a potential hypolipidemic agent for preventing and treating hyperlipidemia [51].

A previous study has validated the traditional use of M. loriformis extract for diabetes mellitus because of its antihyperglycemic activity. $M$. loriformis extract is a potent antidiabetic agent, which improves blood glucose level in diabetic patients $[12,39]$. At $1 \mathrm{mg} / \mathrm{mL}$, the respective plant extract markedly inhibited $34.63 \%$ of glycation in fructosemediated nonenzymatic glycation at early week [39]. $M$. loriformis extracts demonstrated moderate pancreatic $\alpha$-amylase and intestinal $\alpha$-glucosidase, maltase, and sucrose inhibition with $\mathrm{IC}_{50}$ values of $0.86,3.43$, and $3.46 \mathrm{mg} / \mathrm{mL}$, respectively. In the study, $M$. loriformis extracts had $12.2 \mathrm{mg} /$ $\mathrm{g}$ of total phenolic content followed by flavonoids $4.34 \mathrm{mg} / \mathrm{g}$ and condensed tannins at $100.7 \mathrm{mg} / \mathrm{g}$, which indicates the potential of $M$. loriformis antidiabetic properties. Phenolic, mainly flavonoids, rich extracts have better $\alpha$-glucosidase inhibitory activity that controls blood glucose and type 2 diabetes [73-75].

\section{Toxicity}

Acute toxicity study for 14 days after oral administration of M. loriformis extract at $5000 \mathrm{mg} / \mathrm{kg}$ body weight did not demonstrate any signs of mortality [13]. Tested rats similarly demonstrated general behavior and physiological activities in comparison to the control group. No abnormalities and pathological changes were observed in terms of size and color of internal rat organs suggesting that the $M$. loriformis extract was not toxic at $5000 \mathrm{mg} / \mathrm{kg}$. However, the water extract of $M$. loriformis decreased T- and B-cell proliferation with or without mitogen [24]. Hence, further studies should be carried out on their toxicity effect on nonspecific cellular immune responses to justify the safety of $M$. loriformis extract in humans.

\section{Conclusions and Future Perspectives}

It is well documented in the literature that the polar extract of the whole plant of $M$. loriformis is the most studied part of this plant. Biological activity of polar extracts is associated with their phenolic compounds. Phenolic compounds, which are synthesized by plants for their own protection against pests, could also be used in humans as an antioxidant. Antioxidant has many biological functions in the human body to enable preventing various diseases. Polar extracts of $M$. loriformis possess a wide range of pharmacological actions, such as antioxidant, antimutagenicity, anticancer, anti-inflammatory, immunomodulatory effects, antipyretic, analgesic, antimicrobial, antibacterial, and gastroprotective activity that support its folk use.

Although several reports might justify the ethnomedicinal use of $M$. loriformis, the existing data are insufficient, and there is a need for further in vitro and in vivo pharmacological models, as well as clinical studies. Yet, only a few acute toxicology studies report polar extracts of this plant. Future consideration should be on subacute and chronic toxicity tests for other polarity extracts of this plant as well (semipolar and nonpolar). Because M. loriformis plants traditionally consume fresh or decoction with water, it is essential to ensure that the intake is kept below tolerance levels. Safer dosage limits for human consumption and effects on the target organs toxicity such as lung, liver, kidney, and heart should be clarified and clearly evaluated.

To date, knowledge on the phytochemical constituents of M. loriformis is limited. Therefore, further research is needed for phytochemistry analysis of $M$. loriformis for better understanding of the mechanisms underlying the bioactive constituents and its bioactivity. Future research should employ sophisticated chromatography and spectroscopic techniques such as supercritical fluid chromatography, LCNMR, and LC-QTOF over the standard analysis methods.

Moreover, consideration should be given to extraction techniques including extraction time, temperatures, and solvent type and ratio to optimize extraction yields in addition to enhancing the interest components. Hence, a systematic design of optimization process using effective tools such as response surface methodology and mixture design may offer many advantages over conventional optimization method, which is tedious, lacks data evaluation, and is expensive. Future research should put emphasis on biological studies on a particular part of the plant such as leaves, flowers, and roots because the existing data focus on the whole part of $M$. loriformis.

It is hoped that this present review bridges the gap between traditional use and pharmacological studies of M. loriformis, hence, providing basic information with significant directions for future researchers in developing therapeutic agents and product applications based on this plant.

\section{Data Availability}

The data used to support the findings of this study are included within the article.

\section{Conflicts of Interest}

The authors declare that there are no conflicts of interests regarding the publication of this paper.

\section{Acknowledgments}

The authors would like to acknowledge financial support for the publication of this manuscript by the Research Management Centre of The Universiti Putra Malaysia.

\section{References}

[1] L. S. Nelson, M. Loh, and J. Perrone, "Assuring safety of inherently unsafe medications: the FDA risk evaluation and mitigation strategies," Journal of Medical Toxicology, vol. 10, no. 2, pp. 165-172, 2014.

[2] D. J. Newman and G. M. Cragg, "Natural products as sources of new drugs over the nearly four decades from 01/1981 to 09/ 2019," Journal of Natural Products, vol. 83, no. 3, pp. 770-803, 2020. 
[3] R. J. Molyneux, S. T. Lee, D. R. Gardner, K. E. Panter, and L. F. James, "Phytochemicals: the good, the bad and the ugly?," Phytochemistry, vol. 68, no. 22-24, pp. 2973-2985, 2007.

[4] I. S. Che Sulaiman, S. Sukhi, and A. Mohamad, "Roles of phytochemicals in the prevention and treatment of various diseases," Phytochemistry, vol. 2, pp. 147-164, 2018.

[5] B. Poonthananiwatkul, R. H. M. Lim, R. L. Howard, P. Pibanpaknitee, and E. M. Williamson, "Traditional medicine use by cancer patients in Thailand," Journal of Ethnopharmacology, vol. 168, pp. 100-107, 2015.

[6] O. Neamsuvan, N. Madeebing, L. Mah, and W. Lateh, "A survey of medicinal plants for diabetes treating from Chana and Nathawee district, Songkhla province, Thailand," Journal of Ethnopharmacology, vol. 174, pp. 82-90, 2015.

[7] W. Jiratchariyakul, P. Moongkarndi, H. Okabe, and A. W. Frahm, "Investigation of anticancer components from Murdannia loriformis (hassk.) Rolla Rao et Kammathy," Thai Journal of Pharmacology, vol. 5, no. 1, pp. 10-20, 1998.

[8] W. Jiratchariyakul and T. Kummalue, "Experimental Therapeutics in Breast Cancer Cells," in Breast Cancer-Current and Alternative Therapeutic Modalities, IntechOpen, London, UK, 2011.

[9] MT Chomnawang, S Surassmo, VS Nukoolkarn, and W Gritsanapan, "Antimicrobial effects of Thai medicinal plants against acne-inducing bacteria," Journal of Ethnopharmacology, vol. 101, no. 1-3, pp. 330-3, 2005.

[10] Y. Intiyot, T. Kinouchi, K. Kataoka et al., "Antimutagenicity of Murdannia loriformis in the Salmonella mutation assay and its inhibitory effects on azoxymethane-induced DNA methylation and aberrant crypt focus formation in male F344 rats," Journal of Medical Investigation, vol. 49, pp. 25-34, 2002.

[11] W. Jiratchariyakul, M. Vongsakul, L. Sunthornsuk et al., "Immunomodulatory effect and quantitation of a cytotoxic glycosphingolipid from Murdannia loriformis," Journal of Natural Medicines, vol. 60, no. 3, pp. 210-216, 2006.

[12] K. Kittipongpittaya, W. Rungjang, and S. Klomlab, "Investigation of using Beijing grass extract as a natural antioxidant in edible oil," KMUTNB International Journal of Applied Science and Technology, vol. 9, no. 1, pp. 45-52, 2016.

[13] P Kunnaja, SP Wongpalee, and A Panthong, "Evaluation of anti-inflammatory, analgesic, and antipyretic activities of the ethanol extract from Murdannia loriformis (Hassk.) Rolla Rao et Kammathy," BioImpacts: BI, vol. 4, no. 4, pp. 183-189, 2014.

[14] P. Kunnaja, N. Chiranthanut, P. Kunanusorn et al., "Evaluation of gastroprotective potential of the ethanol extract from Murdannia loriformis in rats," International Journal of Applied Research in Natural Products, vol. 8, no. 1, pp. 34-41, 2015.

[15] H. Mori, Y. Yamada, T. Kuno, and Y. Hirose, "Aberrant crypt foci and $\beta$-catenin accumulated crypts; significance and roles for colorectal carcinogenesis," Mutation Research/Reviews in Mutation Research, vol. 566, no. 3, pp. 191-208, 2004.

[16] U. Vinitketkumnuen, T. Chewonarin, P. Dhumtanom, N. Lertprasertsuk, and C. P. Wild, "Aflatoxin-albumin adduct formation after single and multiple doses of aflatoxin B1 in rats treated with Thai medicinal plants," Mutation Research/ Fundamental and Molecular Mechanisms, vol. 428, no. 1-2, pp. 345-351, 1999.

[17] S. Z. Y. Shu, "Murdannia Royle, Ill. Bot. Himal. Mts. 1: 403. 1840, nom. cons," Flora of China, vol. 24, pp. 25-31, 2000.

[18] N. A. Hamzah, "Khasiat Sebalik Rumput Beijing," Sinar Harian. http://www.sinarharian.com.my/mobile/edisi/ melaka-ns/khasiat-sebalik-rumput-beijing-1.628466.
[19] “Angel Grass Aka Siti Khatijah (Beijing Grass)," 2018. https:// mymesraherbs.wordpress.com/2018/02/06/angel-grass-akasiti-khatijah-beijing-grass/.

[20] "The Plant List," 2013. http://www.theplantlist.org.

[21] B. Y. J. K. Morton, "A revision of the genus Aneilema R. Brown (Commelinaceae) with a cytotaxonomic account of the West African species," Botanical Journal of the Linnean Society, vol. 59, no. 1810, pp. 431-481, 1966.

[22] C. Prasitpuriprecha, B. Sripanidkulchai, V. Lulitanond, and J. Saguansermsri, "Studies on the uilization of medicinal plants as immunomodulators in Ubon Ratchathani Province, Thailand," KKU Research Journal, vol. 10, no. 1, pp. 31-41, 2005.

[23] N Cheeptham and G. H. Towers, "Light-mediated activities of some Thai medicinal plant teas," Fitoterapia, vol. 73, no. 7-8, pp. 651-62, 2002.

[24] K. Punturee, W. Kasinrerk, C. P. Wild, and U. Vinitketkumnuen, "Immunomodulatory effects of Thai medicinal plants on the mitogen stimulated proliferation of human peripheral blood mononuclear cells in vitro," Chiang Mai Med Bull, vol. 44, no. 1, pp. 1-12, 2005.

[25] K. C. Chinsembu, "Diabetes mellitus and nature's pharmacy of putative antidiabetic plants," Journal of Herbal Medicine, vol. 15, Article ID 100230, 2019.

[26] C. Pinitsoontorn, S. Suwantrai, and P. Boonsiri, "Antioxidant activity and oxalate content of selected Thai herbal teas," KKU Research Journal, vol. 17, no. 1, pp. 162-168, 2012.

[27] Y. Intiyot, "Effects of Murdannia loriformis extract on azoxymethane-induced DNA-adducts and aberrant crypt foci in rat colons," M.S. thesis, Chiang Mai University, Chiang Mai, Thailand, 1998.

[28] P. Nootim, N. Kapol, W. Bunchuailua, P. Poompruek, and P. Tungsukruthai, "Current state of cancer patient care incorporating Thai traditional medicine in Thailand: a qualitative study," Journal of Integrative Medicine, vol. 18, no. 1, pp. 41-45, 2020.

[29] "Rumput cina penawar kanser." http://ubatkanser.my/ rumput-cina-penawar-kanser/, 2018.

[30] "Rumput Cina-Beijing Grass Plant (Murdannia loriformis)." http://spmodels.net/nature/rumput-cina-beijing-grass-plantmurdannia-loriformis/, 2018.

[31] ChinaHao, "Angel Grass Water Surplus Moisturizing Cream," 2016. https://www.chinahao.com/product/547684277665/ Emulsion.

[32] Athena (Guangzhou) Cosmetics Manufacturer, "Skin Care Angel Grass Burst Water Ice Dew Gel,” 2018. https:// athenaoem.en.made-in-china.com/product/yKvxdJmlgApW/ China-OEM-Skin-Care-Angel-Grass-Burst-Water-Ice-DewGel.html.

[33] T. Care, "Pak King Capsules Angel Grass (Beijing Grass)," 2018. http://en.thaicare100.com/ya-pak-king?search=angel grass.

[34] C. S. na Ayuthya, "Beiing Grass-Murdannia loriformis Capsules,” 2017. http://eurasiatrade.ch/English/Redtones/ KLOandothers-singleproducts/ MurdannialoriformisBejingGrass.htm.

[35] A. Kaewnoi, S. Duanyai, K. Tongthong et al., "Preparation of Beijing Grass tablets from spray-dried powder of juice of Murdannia loriformis (Hassk.) Rolla Rao et Kammathy," Journal of Innovations in Pharmaceutical and Biological Sciences, vol. 5, no. 3, pp. 11-25, 2018.

[36] Mistine, "Healthy Tea Compound Murdannia loriformis Infusion Beijing/Angel Grass," 2016. https://mistine.ecrater. $\mathrm{com} / \mathrm{p} / 21871686 / 100 \mathrm{x}$-healthy-tea-compound-murdannia. 
[37] T. Parametthanuwat, S. Aichayawanich, and J. Wongsa, "Development of herbal gummy jelly with brown sugar and Angel grass extract," in Proceedings of the 9th International Conference on Sciences, Technology and Innovation for Sustainable Well-Being, Vienna, Austria, June 2017.

[38] Watson, "Green Tea Seed Moisturizing Mask Angel Grass," 2018. https://www.yoycart.com/Product/549374168536/\% $0 \mathrm{~A}$ (Watson)the.

[39] S. Adisakwattana, P. Jiphimai, P. Prutanopajai, B. Chanathong, S. Sapwarobol, and T. Ariyapitipan, "Evaluation of $\alpha$-glucosidase, $\alpha$-amylase and protein glycation inhibitory activities of edible plants," International Journal of Food Sciences and Nutrition, vol. 61, no. 3, pp. 295-305, 2010.

[40] W. Jiratchariyakul, "A steroidal glucoside from Murdannia loriformis (Hassk.) Rolla Rao et Kammathy,” Thai Journal of Pharmacology, vol. 3, pp. 31-39, 1996.

[41] B. Techaratanakrai, S. Chaiwanichsiri, and K. Laohasongkram, "Effects of infusion temperature and time on antioxidant activity of herbal infusions," in Proceedings of the 33rd Congress on Science and Technology of Thailand, Nakhon Si Thammarat, Thailand, October 2017.

[42] K. Pongsathorn, "Determination of antioxidant property from some medicinal plant extracts from Thailand," African Journal of. Biotechnology, vol. 11, no. 45, pp. 10322-10327, 2012.

[43] P. N. Phatthalung, S. Chusri, and S. P. Voravuthikunchai, "Thai ethnomedicinal plants as resistant modifying agents for combating Acinetobacter baumannii infections," BMC Complementary Medicine and Therapies, vol. 12, 2012.

[44] V. Tzin and G. Galili, "New insights into the shikimate and aromatic amino acids biosynthesis pathways in plants," Molecular Plant, vol. 3, no. 6, pp. 956-972, 2010.

[45] S. Koontongkaew, K. Suriyawong, and W. Siengboon, "Effects of Murdannia loriformis extracts on cancer cell growth," in Proceedings of the 2nd Meeting of IADR Pan Asian Pacific Federation (PAPF) and the 1st Meeting of IADR Asia/Pacific Region, Pathumthani, Thailand, July 2009.

[46] T. Rearungchom, "Possible mechanism of inhibition of aflatoxin-B1 mutagenesis by Thai medicinal plants: Murdannia loriformis and Alpinia galanga," M.S. Thesis, Chiang Mai University, Chiang Mai, Thailand, 1993.

[47] H. K. Lichtenthaler and A. R. Wellburn, "Determinations of total carotenoids and chlorophylls a and b of leaf extracts in different solvents," Biochemical Society Transactions, vol. 11, no. 5, pp. 591-592, 1983.

[48] S. Somja, "Anti-inflammatory, analgesic and antipyretic activities of ethanol extract from Murdannia loriformis (Hassk.) Rolla Rao et Kammathy," M.S. thesis, Chiang Mai University, Chiang Mai, Thailand, 2005.

[49] S. Limsuwan, S. Subhadhirasakul, and S. P. Voravuthikunchai, "Medicinal plants with significant activity against important pathogenic bacteria," Pharmaceutical Biology, vol. 47, no. 8, pp. 683-689, 2009.

[50] M. T. Chomnawang, S. Surassmo, K. Wongsariya, and N. Bunyapraphatsara, "Antibacterial activity of Thai medicinal plants against methicillin-resistant Staphylococcus aureus," Fitoterapia, vol. 80, no. 2, pp. 102-104, 2009.

[51] S. Adisakwattana, J. Intrawangso, A. Hemrid, B. Chanathong, and K. Mäkynen, "Extracts of edible plants inhibit pancreatic lipase, cholesterol esterase and cholesterol micellization, and bind bile acids," Food Technology and Biotechnology, vol. 50, no. 1, pp. 11-16, 2012.

[52] R. L. Schleicher, L. F. McCoy, C. D. Powers, M. R. Sternberg, and C. M. Pfeiffer, "Serum concentrations of an aflatoxinalbumin adduct in the national health and nutrition examination survey (NHANES) 1999-2000," Clinica Chimica Acta, vol. 423, pp. 46-50, 2013.

[53] P. A. Egner, S. J. Gange, P. M. Dolan, J. D. Groopman, A. Muñoz, and T. W. Kensler, "Levels of aflatoxin-albumin biomarkers in rat plasma are modulated by both long-term and transient interventions with oltipraz," Carcinogenesis, vol. 16, no. 8, pp. 1769-1773, 1995.

[54] H. Moulahoum, B. M. A. Boumaza, M. Ferrat et al., "Aberrant crypt foci are regionally affected by zinc treatment in a 1,2dimethylhydrazine induced colon carcinogenesis model," Journal of Trace Elements in Medicine and Biology, vol. 47, pp. 21-30, 2018.

[55] U. Vinitketkumnuen, W. Charoenkunathum, P. Kongtawelert, N. Lertprasertsuke, P. Picha, and T. Matsushima, "Antimutagenicity and DT-diaphorase inducer activity of the Thai medicinal plant, Murdannia loriformis," Journal of Herbs, Spices \& Medicinal Plants, vol. 4, no. 1, pp. 45-52, 1996.

[56] S. Danson, T. H. Ward, J. Butler, and M. Ranson, "DT-diaphorase: a target for new anticancer drugs," Cancer Treatment Reviews, vol. 30, no. 5, pp. 437-449, 2004.

[57] J. González-Gallego, M. V. García-Mediavilla, S. SánchezCampos, and M. J. Tuñón, "Anti-inflammatory and immunomodulatory properties of dietary flavonoids," Polyphenols in Human Health and Disease, vol. 1, pp. 435-452, 2014.

[58] A. J. León-González, C. Auger, and V. B. Schini-kerth, "Prooxidant activity of polyphenols and its implication on cancer chemoprevention and chemotherapy," Biochemical Pharmacology, vol. 98, no. 3, pp. 371-380, 2015.

[59] I. S. Che Sulaiman, Design and optimization of nanoemulsion formulation containing extract of Clinacanthus nutans Lindau leaves for cosmeceutical application, $\mathrm{PhD}$ Thesis, Universiti Putra Malaysia, Serdang, Selangor, 2017.

[60] I. S. Che Sulaiman, M. Basri, H. R. Fard Masoumi et al., "Effects of temperature, time, and solvent ratio on the extraction of phenolic compounds and the anti-radical activity of Clinacanthus nutans Lindau leaves by response surface methodology," Chemistry Central Journal, vol. 11, no. 1, pp. 1-11, 2017.

[61] P. G. Mateus, V. G. Wolf, M. S. Borges, and V. F. Ximenes, Quercetin: Prooxidant Effect and Apoptosis in Cancer, Elsevier B.V., Amsterdam, Netherlands, 1st edition, 2018.

[62] E. Rayburn, "Anti-inflammatory agents for cancer therapy," Molecular and Cellular Pharmacology, vol. 1, no. 1, pp. 29-43, 2009.

[63] L. J. Hofseth and L. Ying, "Identifying and defusing weapons of mass inflammation in carcinogenesis," Biochimica et Biophysica Acta (BBA)-Reviews on Cancer, vol. 1765, no. 1, pp. 74-84, 2006.

[64] A. C. Archer, S. P. Muthukumar, and P. M. Halami, "Antiinflammatory potential of probiotic Lactobacillus spp. on carrageenan induced paw edema in Wistar rats," International Journal of Biological Macromolecules, vol. 81, pp. 530-537, 2015.

[65] J. P. Jaismy, S. L. Manju, K. R. Ethiraj, and G. Elias, “Safer antiinflammatory therapy through dual COX-2/5-LOX inhibitors: a structure-based approach," European Journal of Pharmaceutical Sciences, vol. 121, no. 2017, pp. 356-381, 2018.

[66] K. Kunnaja, P. Warunee, K. Punturee, A. Siriphun, and T. Chatboonward, "Inhibition of nitric oxide production and COX-2 protein expression in LPS-stimulated RAW 264.7 cells by the hexane fraction of Murdannia loriformis," Journal of Associated Medical Sciences, vol. 52, no. 2, pp. 103-111, 2019. 
[67] R. Marroquin-Segura, M. Flores-Pimentel, R. CarreónSánchez et al., "The effect of the aqueous extract of Helietta parvifolia A. Gray (Rutaceae) stem bark on carrageenan-induced paw oedema and granuloma tissue formation in mice," Journal of Ethnopharmacology, vol. 124, no. 3, pp. 639-641, 2009.

[68] B. V. Owoyele and A. O. Bakare, "Analgesic properties of aqueous bark extract of Adansonia digitata in wistar rats," Biomedicine \& Pharmacotherapy, vol. 97, pp. 209-212, 2018.

[69] T. Kaewkod, K. Tragoolpua, and Y. Tragoolpua, "Encapsulation of Artocarpus lacucha Roxb. Extract in alginate chitosan nanoparticles for inhibition of methicillin resistant Staphylococcus aureus and bacteria causing skin diseases," Chiang Mai Journal of Science, vol. 43, no. 5, pp. 946-958, 2016.

[70] A. R. S. Ribeiro, P. B. F. Diniz, M. S. Pinheiro et al., "Gastroprotective effects of thymol on acute and chronic ulcers in rats: the role of prostaglandins, ATP-sensitive $\mathrm{K}+$ channels, and gastric mucus secretion," Chemico-Biological Interactions, vol. 244, pp. 121-128, 2016.

[71] A. K. Ramírez-Jiménez, M. E. Tejero, F. León-Galván, G. Loarca-Piña, and G. Loarca-Piña, "Potential role of bioactive compounds of Phaseolus vulgaris L. on lipid-lowering mechanisms," Food Research International, vol. 76, no. P1, pp. 92-104, 2015.

[72] R. Urbatzka, S. Freitas, A. Palmeira et al., "Lipid reducing activity and toxicity profiles of a library of polyphenol derivatives," European Journal of Medicinal Chemistry, vol. 151, pp. 272-284, 2018.

[73] Y. Cai, L. Wu, X. Lin, X. Hu, and L. Wang, "Phenolic profiles and screening of potential $\alpha$-glucosidase inhibitors from Polygonum aviculare L. leaves using ultra-filtration combined with HPLC-ESI-qTOF-MS/MS and molecular docking analysis," Industrial Crops and Products, vol. 154, Article ID 112673, 2020.

[74] M. Alongi, J. María Frías Celayeta, R. Vriz et al., "In vitro digestion nullified the differences triggered by roasting in phenolic composition and $\alpha$-glucosidase inhibitory capacity of coffee," Food Chemistry, vol. 342, Article ID 128289, 2020.

[75] R. Giacco, G. Costabile, G. Fatati et al., "Effects of polyphenols on cardio-metabolic risk factors and risk of type 2 diabetes. A joint position statement of the diabetes and nutrition study group of the Italian society of diabetology (SID), the Italian association of dietetics and clinical nutrition (ADI) and the Italian association of medical diabetologists (AMD)," Nutrition, Metabolism and Cardiovascular Diseases, vol. 30, no. 3, pp. 355-367, 2020. 